\title{
Renal Cell Carcinoma
}

National Cancer Institute

\section{Source}

National Cancer Institute. Renal Cell Carcinoma. NCI Thesaurus. Code C9385.

A carcinoma arising from the renal parenchyma. There is a strong correlation between cigarette smoking and the development of renal cell carcinoma. The clinical presentation includes : hematuria, flank pain and a palpable lumbar mass. A high percentage of renal cell carcinomas are diagnosed when an ultrasound is performed for other purposes. Radical nephrectomy is the standard intervention procedure. Renal cell carcinoma is generally considered to be resistant to radiation treatment and chemotherapy. 